\title{
A Framework for Part Cost Estimation Based on Feature Recognition
}

\author{
LI Weiyu \&ZHANG Xu \\ Beijing Institute of Technology, Beijing, China
}

\begin{abstract}
Manufacturability analysis is the key approach of implementing concurrent engineering. Its major objective is to analyze the factors which might affect the following processes with consideration of manufacturing resources. It can assist the designers to make the correct decision during the early design stage and avoid some downstream manufacturing issues. In this paper, a method for part cost estimation in terms of feature recognition has been presented. Part cost is determined by shape complexity, product precision, and tooling process. Combined with feature recognition result and the manufacturing resource information, this method can obtain the part cost by calculating the machining time for each feature.
\end{abstract}

Keywords: Feature recognition; Manufacturability analysis; Part cost estimation

\section{INTRODUCTION}

Manufacturability analysis is the key approach of implementing concurrent engineering. Its major objective is to analyze the factors which might affect the following processes with consideration of manufacturing resources. It can help the designers to make the right decision at the design stage, and hence save the development time and improve the product quality (Hongjun Liu et al. 2009).

Several Manufacturability analysis methods have been developed over the past decades. Currently, the measure of manufacturability can be classified into three types: binary measure, qualitative measure and time\&cost measure (Gupta S K et al. 1997). Among these, part cost estimation is one aspect which attracted many researchers attention. Its main idea is to estimate the part cost at the early stage of the development cycle in order to achieve the following goals: (Ou-Yang C, Lin T S. 1997)

a) To find the portion of a part which might cause a high manufacturing cost.

b) To allow the designers estimate the alternative cost for comparative design cost.

In this paper, we proposed a part cost estimate method which is based on feature recognition. It extracted feature information from the part model by employing rules-based feature-recognition algorithms, and then performed the part cost estimation using manufacturing resources data.

\section{BACKGROUND}

Actually, manufacturability analysis is a huge and complicated work, covering various aspects of manufacturing knowledge, such as material selection, operation decision and progress sequence. Most parts could not be fabricated by a single operation, so there are many kinds of manufacturability analysis methods responding to the different manufacturing operations.

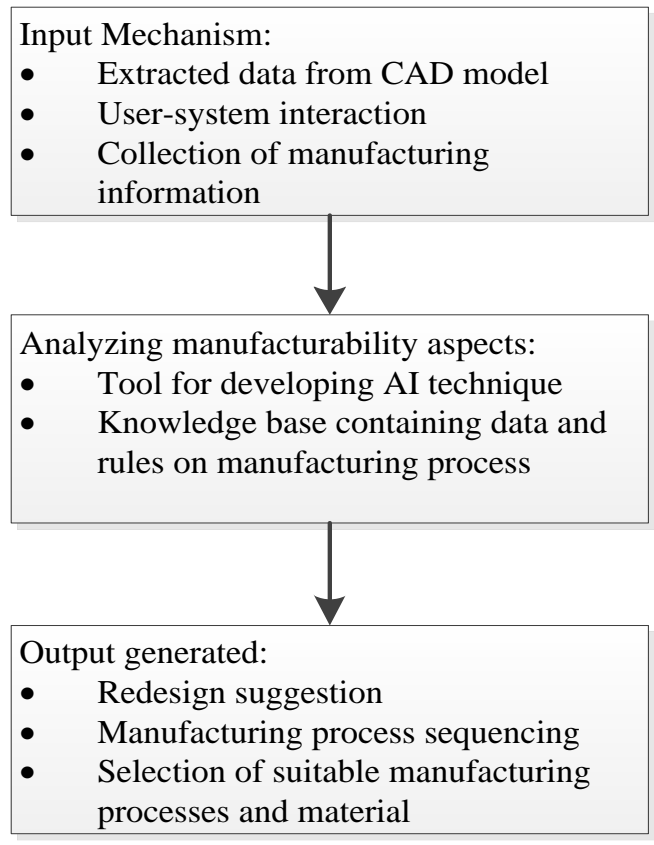

Figure 1 The process of manufacturability analysis (Gupta S K et al. 1997) 
The published literatures on manufacturability analysis have made many researches. Generally, the progress of manufacturability can be divided into three segments:

1. Input mechanism

2. Analyzing manufacturability aspects

3. Output generated

Figure 1 shows the process of manufacturability analysis.

Part cost estimation (PCE) is a direct and effective means of the manufacturing analyzing. In the traditional develop cycle, however, PCE is not performed until the whole process planning work have been finished. If the cost exceeds the budget, part had to be transferred back to the design apartments to redesign until it meets the budget, which add some unnecessary irritations among the different development apartments. As a result, it will lengthen development time, lower the productivity and increase the product cost.

Research results suggest that over $70 \%$ product cost is determined at the design stage (Dewhurst P, Boothroyd G. 1994). It means that if the designers discover the issues which might appear in the subsequent progress and made the correct decision at the early design stage, the lead time to markets could be reduced and the quality could be improved. In order to tackle this issue, PCE is performed at the design stage. Figure 2 shows the two different ways.

Various kinds of PCE models have been developed. An Neural Networks (NN) system was developed by Zhaohui Wang (2004).This system allows users to input the part information through PCE interface at the beginning, and then it will search the database to find whether there is a corresponding NN model. If there is one, the system can calculate the part cost with this model directly. If not, the system need to train the $\mathrm{NN}$ with the original data (sample data) to obtain the correct NN Model in order to estimate the part cost.

a method based on mathematical fuzzy close-indegree developed by Zhixing $\mathrm{Xu}$ (2000). The main objective of this method is to estimate the part which is similar to one whose cost has been calculated. This method first extracts the factors affecting the cost of parts, and membership of the part corresponding to these factors proposed by knowledge system according to the relationship between these factors. according to the definition of nearness of new parts can be calculated with the various parts of the existing Closeness, Finally, according to the degree of similarity of size close to the new parts can be discharged with the existing parts, using exponential smoothing prediction method can predict the cost of new parts.

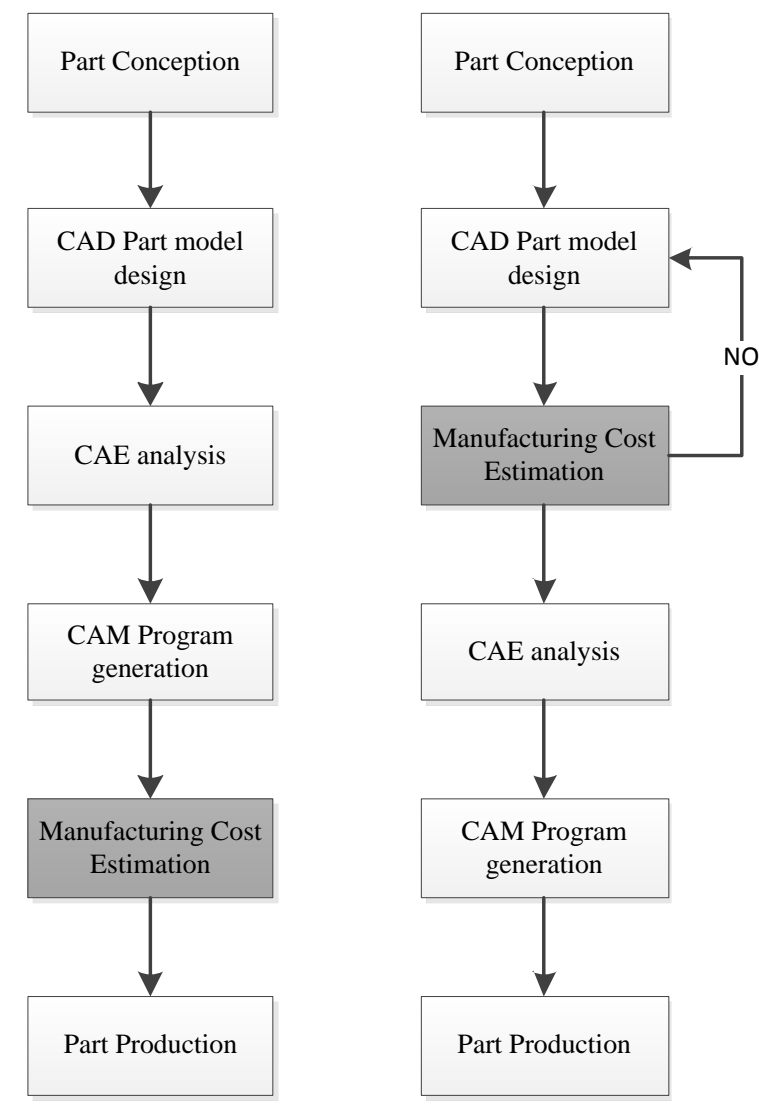

Figure 2 Two different ways of part development

Dewhurst and Boothoyd (1988) developed a cost model for machined parts. This model requires only limited information(such as machining time, rate for machines and operators, and types of tooling materials) to estimate machining cost. It seems that most of these data can be obtained during the design stage except for the precise machining time. Therefore, this model can be used in the early cost estimation process if an approach to measure the machining time can be included.

From the above literature review, we can see the researchers have developed a lot of calculation methods and models to assess the cost of the product. Consolidated on the basis of existing research results, this paper presents an interactive method for calculating the cost of part based on feature-recognition. This method will calculate the cost based on the characteristics of the geometric parameters and associated PMI information, combined with data obtained from manufacturing resource, helping the designers find the issues affecting product cost in the early stage and improving production efficiency.

\section{SYSTEM FRAMEWORK}

Figure 3 shows the framework of proposed system. There are three main modules: feature-recognition 
module, analyzing module and cost estimate module.

Feature-recognition module extract features and properties associated with them from input CAD part model and construct the feature information model. Figure 4 shows the feature information model of the proposed system, and it is composed of part basic information, dimension information, feature basic information and geometry topology information.

Part basic information incorporates part-level information, such as name, ID, material and amount of the part. These can be inherited from the part design model.

Feature basic information guarantees the feature exist in the model features as a unique identity.

Dimension information incorporates design dimensions and specifications, such as roughness, precision and datum.

Geometry and topology information corporates a set of faces and related information.

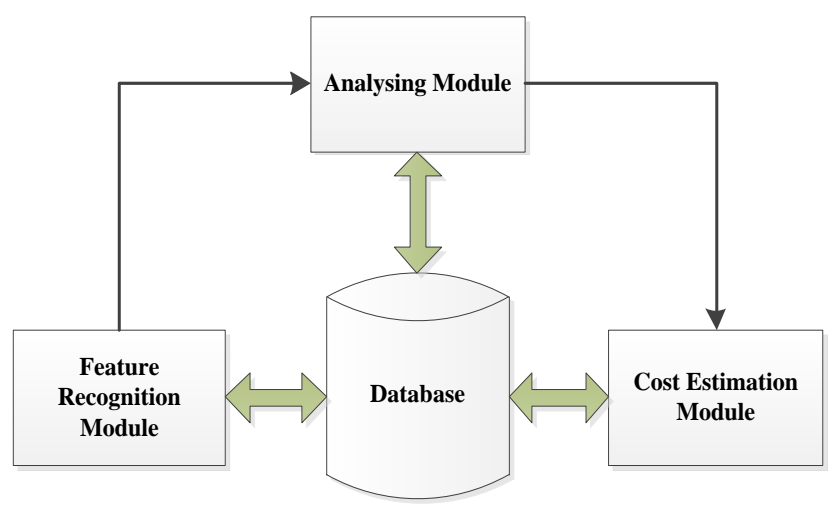

Figure 3 The framework of the cost estimation system

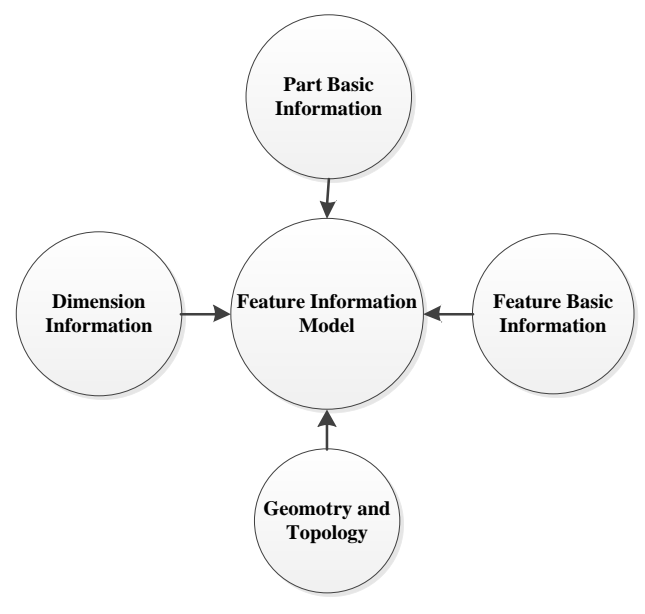

Figure 4 The Structure of Feature Information Model

The major module of the proposed work is the analyzing module. Its main function can be divided into two sub-functions. The first one is to perform a decision-making work for operations based on the feature types. In other words, it will build a map between the feature and the machining method. Table 1 demonstrates some basic features and corresponding machining methods. The knowledge used in this progress can be obtained from the database integrated in the system. The second function is to establish the machining feature information model based on the feature information model. Some parameters need to be determined, such as volume of material removed, cutting speed, tool size etc.

The function of cost estimate module is to calculate the cost of the part. It first compute the machining time for features and then transform the machining time into cost using manufacturing resources data. These data can be obtained from handbooks and statistics of enterprises.

\section{COST ESTIMATION PROCESS}

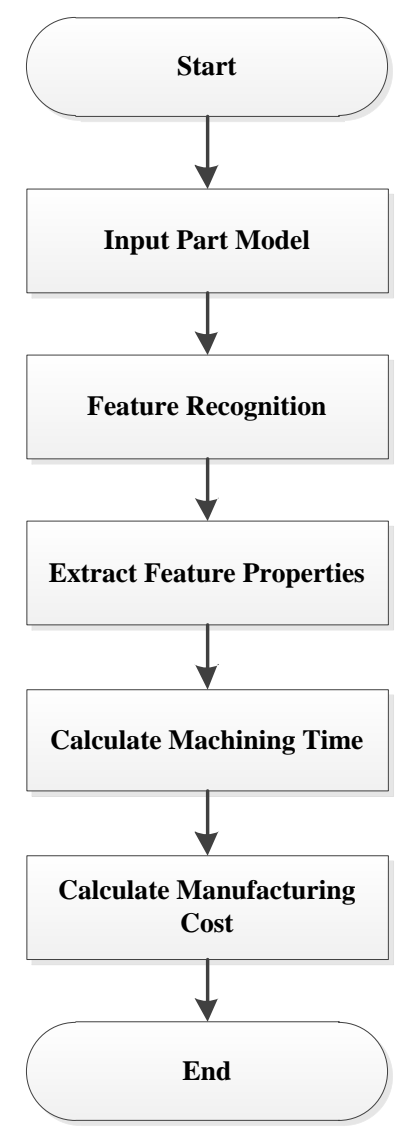

Figure 5 Process of cost estimation

Table 1 Machining method corresponding to Feature

\begin{tabular}{|l|l|l|}
\hline $\begin{array}{l}\text { Feature } \\
\text { Types }\end{array}$ & Machining Methods & Topology Characters \\
\hline Hole & Drilling & Cone without concave side \\
\hline Plain & Milling & Plain without concave side \\
\hline Surface & Milling, Turning & Surface without concave side \\
\hline Slot & Milling & Plain with concave side \\
\hline
\end{tabular}

Based on the proposed framework, Figure 5 shows the flowchart of the cost estimation process. The process can be described as follows: 
Step 1: Extract the geometry and topology information and product manufacturing information (PMI) from the CAD files (referred to PRO Engineer files).

Step 2: Set feature type information and run the feature recognition work.

Step 3: Create the machining feature model based on the feature recognition result and related properties.

Step 4: Calculate machining time for every machining feature

1) Calculate the cutting time required for each step

$$
\mathrm{T}_{i j}=\frac{V_{i j}}{M R R_{i j}}
$$

Where:

$T_{i j} \quad=$ time required to accomplish the machining step $\mathrm{j}$ of feature $\mathrm{i}$.

$V_{i j} \quad=$ volume removed in machining step $\mathrm{j}$ of feature $\mathrm{i}$.

$M R R_{i j}=$ material removal rate of machining step $\mathrm{j}$ of feature $\mathrm{i}$.

2) Calculate manufacturing cost for each step

$$
C_{i j}=M_{k} \mathrm{~T}_{i j}+S_{k}
$$

Where:

$C_{i j}=$ the machining cost of the machining step $\mathrm{j}$ of feature I

$M_{i j}=$ unit time cost of machine k

$S_{k} \quad=$ set-up cost of machine k

3) Calculate manufacturing cost for the machining feature.

$$
F C_{i}=\sum_{j}^{n} C_{i j}
$$

Where:

$F C_{i}=$ machining cost of feature $\mathrm{i}$.

Step 5: Calculate the manufacture cost for the whole part.

$$
P C=\sum_{i}^{n} F C_{i}
$$

Where:

$P C=$ machining cost of the part.

\section{SYSTEM IMPLEMENTATION}

This system employs ACIS as its geometry model kernel and InterOp as the interface in order to transform the input CAD files (mainly refer to PRO Engineer files) into SAT files, and perform the analyzing and calculating work presented above.

Manufacturing resource library and knowledge system are built upon SQL SERVER database.
Users can use SQL to add, edit and delete these data according to the actual situation of enterprises. Table 2 shows some information about the machine

Table 2 Machine Information table

\begin{tabular}{|l|l|l|l|}
\hline $\begin{array}{l}\text { Machine } \\
\text { ID }\end{array}$ & $\begin{array}{l}\text { Operation } \\
\text { Type }\end{array}$ & $\begin{array}{l}\text { Maximum } \\
\text { Machine Resolution }\end{array}$ & $\begin{array}{l}\text { Unit Time } \\
\text { Cost }\end{array}$ \\
\hline C001 & Milling & IT7 & 10.00 \\
\hline & Drilling & IT8 & 10.00 \\
\hline M002 & Milling & IT9 & 9.00 \\
\hline D003 & Drilling & IT9 & 9.00 \\
\hline T004 & Turning & IT9 & 9.00 \\
\hline
\end{tabular}

\section{CASE STUDY}

Figure 6 shows a part model with some basic features: a step hole, a through slot and a pocket.

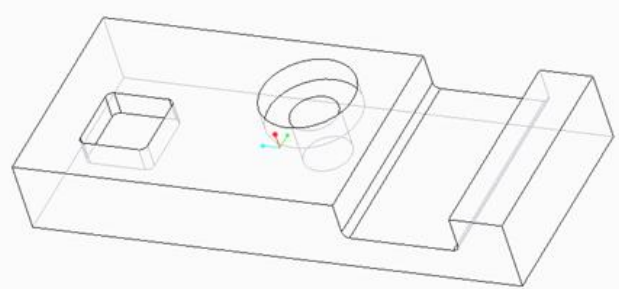

Figure 6 The sample part

Figure 7 shows the result of feature-recognition. It is demonstrated by a tree structure, and every node on the tree presents a feature. The sub-nodes of the nodes is the machining methods. In other words, feature nodes correspond to the feature information model and sub-nodes correspond to the machining feature information model.

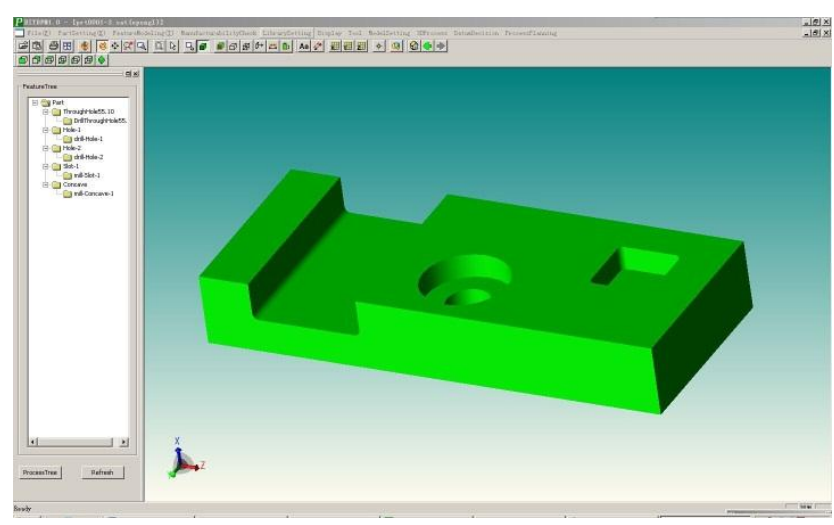

Figure 7 Feature recognition result

Figure 8 shows the result the part cost estimation, which is made up of material cost and machining cost. Material cost can be obtained by setting the type of raw material and the price. Machining cost is 
also demonstrated with a tree, corresponding to the feature tree.

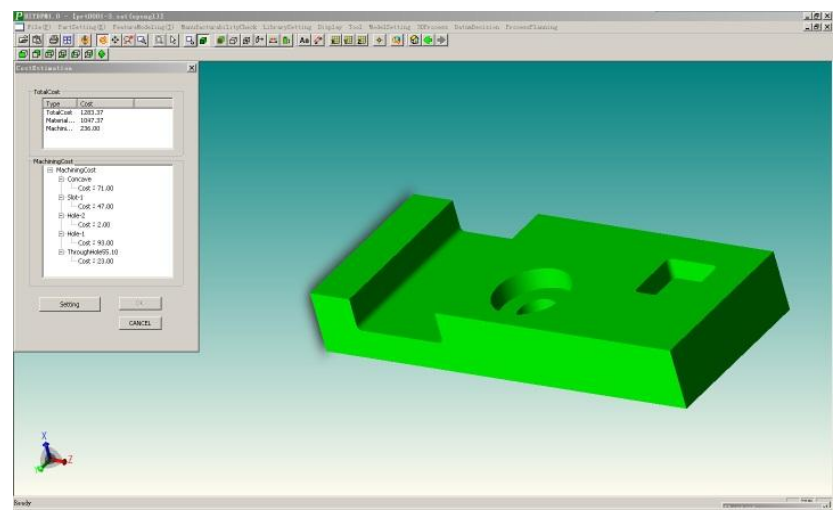

Figure 8 Cost estimation result

We will take the hole feature as a instance to explain the costing progress in detail. Its diameter is $15 \mathrm{~mm}$, depth is $60 \mathrm{~mm}$ and we assume its material is 45 \# steel. The estimation progress as follows:

1) Calculate the removed volume

$$
\begin{aligned}
V & =0.25 \times \pi \mathrm{h} D^{2} \\
& =0.25 \times 3.14 \times 60 \times 15^{2} \\
& =10597.5 \mathrm{~mm}^{3}
\end{aligned}
$$

Where:

$\mathrm{h}=$ the depth of the hole

$\mathrm{D}=$ the diameter of the hole

2) Calculate the machining time

$$
\begin{aligned}
\text { MRR } & =\left(\pi D^{2} / 4\right) \times(\mathrm{f}) \\
& =\left(3.14 \times 15^{2} / 4\right) \times(500) \\
& =88312.5 \mathrm{~mm}^{3} / \mathrm{min} \\
\mathrm{T} & =\frac{V}{M R R} \\
= & \frac{10597.5}{88312.5} \\
= & 0.12 \mathrm{~min}
\end{aligned}
$$

Where:

$\mathrm{f} \quad=$ the feed speed $(\mathrm{mm} / \mathrm{min})$

$M R R=$ Material Removal Rate
Finally, the cost can be estimated based on the cost per unit time for the machine.

\section{CONCLUSION}

Cost estimation is an area which has attracted much attention recently. One major goal of this approach is to identify an overly expensive design during the early stage of the development cycle. A system based on feature recognition is proposed in this paper. It concludes feature recognition module, analyzing module and cost estimation module. The major contributions of this paper are:

(i). A system to support designer for analyzing the manufacturing cost of a part during the early stage is established.

(ii). Feature recognition is adopted in order to extract information from the part model automatically.

\section{REFERRANCES}

[1] Boothroyd, G. 1994. Product design for manufacture and assembly.Computer-Aided Design, 26(7), 505-520.

[2] Dewhurst, P., \& Boothroyd, G. 1988. Early cost estimating in product design. Journal of Manufacturing systems, 7(3), 183-191

[3] Gupta, S. K., Regli, W. C., Das, D., \& Nau, D. S. 1997. Automated manufacturability analysis: a survey. Research in Engineering Design, 9(3), 168-190.

[4] Hongjun Liu, Rong Mo, Qingming Fan, et al, 2009. Research of manufacturability and evaluation methods of parts based on feature under concurrent engineering environment. Application Research of Computers, 26(3).

[5] Ou-Yang C, Lin T S. 1997. Developing an integrated framework for feature-based early manufacturing cost estimation. The international journal of advanced manufacturing technology, 13(9): 618-629.

[6] Zhaohui Wang, Xiuzhuo Fu. 2004. A Method for Estimating Product Mechanic Cost Based on Neural Networks. Mechatronics, 10(3): 53-55.

[7] Zhixing Xu, Yunliang Ding, Jingui Lu. 2000. A study on method of cost prediction of machinery parts based on mathematical fuzzy close-in-degree. Journal of machine design, 5: 7-9. 